\title{
Safety of users in road evacuation: modelling and DSS for demand
}

\author{
F. Russo \& G. Chilà \\ LAST, Laboratory for Transport Systems Analysis Mediterranea, \\ University of Reggio Calabria, Italy
}

\begin{abstract}
The SICURO research project was developed by the Laboratory for Transport Systems Analysis (LAST). The project includes demand analysis for transportation system simulation in emergency conditions. Herein we propose the specification and calibration of generation and distribution with modal choice models. Using SP (Stated Preference) and RP (Revealed Preference) surveys. Software for demand model calibration and estimation is described, with some being used for the proposed model experimentation.
\end{abstract}

Keywords: evacuation conditions, calibration, estimation, demand models.

\section{Introduction}

Several mathematical models have been proposed to simulate travel demand, based on different assumptions and that can be subdivided in relation to different elements [1-3]. The behavioural models used mainly belong to the family of discrete choice models.

Discrete choice models are usually derived under an assumption of utilitymaximizing behaviour (Random Utility Models, RUM). In relation to the distribution of random residuals, different model specifications can be derived and subdivided into two classes: first class, models characterized by probability expressed in closed form, including the GEV (Generalised Extreme Value) models [4] and their specifications, such as the Multinomial Logit and the Nested Logit models; second class, models founded on simulation, including the Multinomial Probit [5] and Mixed Multinomial Logit models [6].

Generally, these models are estimated by maximizing the likelihood function (for the first class) or the simulated likelihood functions (for the second class). 
For this purpose, several estimation packages are available; alternatively numerical procedures may be used [3].

The use of demand models to simulate evacuation involves various problems connected with most cases that may lead to a state of evacuation [7-10]. The events that can generate emergencies, hence evacuation conditions, can be classified by [9, 10]: event kind; effect in time; effect in space; effect in a transport system, which may be on demand, supply or demand-supply interaction.

When a dangerous event occurs, in evacuation conditions, demand models specified and calibrated in ordinary conditions cannot be directly applied for several reasons: multiplicity of decision-makers; definition of choice set; statistical and probabilistic aspects; attributes and parameters. Moreover, in evacuation conditions the analyst must consider possible targets set by the public decision-maker, in order to reduce system management costs, maximise system utility (safety, security) and reduce traffic incidents [11]. Different demand models have to be specified, in relation to event types, which can be classified according to their effects in space and in time. In relation to these effects, several evacuation scenarios can be defined.

In the international literature, one type of natural event analyzed is the hurricane. In the literature on hurricane evacuation, travel demand estimation is usually subdivided into two steps: estimation of total evacuation demand and estimation of departure time [12]. Generally, these steps are developed considering a statistical approach [13]. In some specifications, the decision to evacuate, in the face of an oncoming hurricane, is simulated as a series of binary choices over time [13].

Our paper is structured as follows: we describe a synthesis of the proposed demand model to simulate transport demand in emergency conditions in section 2, the software available to calibrate demand models in section 3 and the DSS to evaluate demand in section 4; our main conclusions are drawn in section 5 .

\section{Demand model estimation for emergency conditions}

In this section the demand model estimation for emergency conditions, considering RP and SP approaches, and the proposed demand model are described. The proposed demand model includes a generation model; a model simulating modal choice jointly with distribution. For the other specifications, we refer to [9].

\subsection{RP and SP approaches}

Demand model estimation becomes a complex problem, particularly when unpredictable events happen and users fail to respect targets set by the public decision-maker. Prediction of user behaviour becomes essential. For this purpose, evacuation trials and SP (stated preference) surveys may be conducted. Such surveys represent the stated behaviour of users in relation to hypothetical 
contexts. During evacuation trials, RP data can be obtained, even if they are affected by the laboratory effect, because each user participating in evacuation trials knows that he/she is not in real danger. Therefore, RP surveys during evacuation trials are a statement of behaviour in evacuation conditions, similar to SP surveys with physical verification. SP surveys allow us to simulate several evacuation scenarios, which can differ in user category, in the effect in space and time of the dangerous event.

SP surveys should be considered as complementary to traditional RP surveys and the combined use of the two can balance reciprocal merits and shortcomings. In terms of demand modelling it is therefore useful to carry out joint calibrations using RP and SP surveys on the same sample or on different samples of users. Random utility models explaining RP and SP choices should be specified separately since their attributes, random residuals variances and, in principle, even functional forms might be different. Possible specifications of the perceived utilities in both models are formalized below [14]:

$$
\begin{array}{cc}
\text { RP model } & \mathrm{U}_{\mathrm{j}}^{\mathrm{RP}}=\mathrm{V}_{\mathrm{j}}^{\mathrm{RP}}+\varepsilon_{\mathrm{j}}^{\mathrm{RP}}=\beta^{\mathrm{T}} \mathrm{X}_{\mathrm{j}}^{\mathrm{RP}}+\eta^{\mathrm{T}} \mathrm{W}_{\mathrm{j}}^{\mathrm{RP}}+\varepsilon_{\mathrm{j}}^{\mathrm{RP}} \\
\text { SP model } & \mathrm{U}_{\mathrm{j}}^{\mathrm{SP}}=\mathrm{V}_{\mathrm{j}}^{\mathrm{SP}}+\varepsilon_{\mathrm{j}}^{\mathrm{SP}}=\beta^{\mathrm{T}} \mathrm{X}_{\mathrm{j}}^{\mathrm{SP}}+\gamma^{\mathrm{T}} \mathrm{Z}_{\mathrm{j}}^{\mathrm{SP}}+\varepsilon_{\mathrm{j}}^{\mathrm{SP}}
\end{array}
$$

where:

$\mathrm{U}_{\mathrm{j}}^{\mathrm{RP}}, \mathrm{U}_{\mathrm{j}}^{\mathrm{SP}}$ are perceived utilities associated with alternative $\mathrm{j}$ in the RP and SP context, respectively;

$\mathrm{X}_{\mathrm{j}}^{\mathrm{RP}}, \mathrm{X}_{\mathrm{j}}^{\mathrm{SP}}$ are vectors of the common attributes relative to the alternative $\mathrm{j}$; these attributes appear in the specification of the RP and SP models with the same coefficients;

$\mathrm{W}_{\mathrm{j}}^{\mathrm{RP}}$ is the vector of the RP specific attributes relative to alternative $\mathrm{j}$;

$Z_{j} \mathrm{SP}$ is the vector of the SP specific attributes relative to alternative $j$;

$\varepsilon_{\mathrm{j}}^{\mathrm{RP}}, \varepsilon_{\mathrm{j}}^{\mathrm{SP}}$ are random residuals of alternative $\mathrm{j}$ in the RP and SP context, respectively;

$\beta, \eta$ and $\gamma$ are the vectors of the unknown parameters to be estimated. Definition of the choice probabilities $\mathrm{p}_{\mathrm{j}}^{\mathrm{RP}}$ and $\mathrm{p}_{\mathrm{j}}^{\mathrm{SP}}$ obviously depends on the assumptions on the distribution of the random vectors $\varepsilon_{j}^{\mathrm{RP}}$ and $\varepsilon_{j}^{\mathrm{SP}}$. Joint calibration is possible introducing a scale factor taking into account the possibility that the variances of the vectors $\varepsilon_{j}^{\mathrm{RP}}$ and $\varepsilon_{\mathrm{j}}^{\mathrm{SP}}$ might be different.

In light of such considerations, SP surveys play a key role, and RP surveys during evacuation trials may be viewed as physically checking SP data.

\subsection{Generation model specification}

Given an emergency scenario, the generation model simulates the mean number of people of category $\mathrm{k}$ to evacuate in the study area in the reference period. In this work we consider:

- the citizen user as the decision-maker;

- a choice set including the alternatives present or otherwise in the reference period and, for people present, the alternatives willing or not to evacuate;

- attributes and parameters related to socio-economic property.

Moreover, we consider the absence of targets.

The model was developed for the following categories: 
- residents within the area (R);

- non-residents who systematically reach the area for work (employees, W), and, in particular, teachers and pupils (school users, S);

- non-residents who occasionally reach the area for shopping or other activities (occasional customers, C);

- weak users (D).

Demand $d_{E, r}(h)$ of those present in the study area in the reference period $h$, can be segmented for categories as:

$$
\mathrm{d}_{\mathrm{E}, \mathrm{r}}(\mathrm{h})=\mathrm{d}_{\mathrm{E}, \mathrm{r}}^{\mathrm{R}}(\mathrm{h})+\mathrm{d}_{\mathrm{E}, \mathrm{r}}^{\mathrm{W}}(\mathrm{h})+\mathrm{d}_{\mathrm{E}, \mathrm{r}}^{\mathrm{C}}(\mathrm{h})+\mathrm{d}_{\mathrm{E}, \mathrm{r}}^{\mathrm{S}}(\mathrm{h})+\mathrm{d}_{\mathrm{E}, \mathrm{r}}^{\mathrm{D}}(\mathrm{h})
$$

with

$\mathrm{d}_{\mathrm{E}, \mathrm{r}}^{\mathrm{R}}(\mathrm{h})$ demand of those present in the resident category;

$\mathrm{d}_{\mathrm{E}, \mathrm{r}}^{\mathrm{W}}(\mathrm{h})$ demand of those present in the employee category;

$\mathrm{d}_{\mathrm{E}, \mathrm{r}}^{\mathrm{C}}(\mathrm{h})$ demand of those present in the occasional customer category;

$\mathrm{d}_{\mathrm{E}, \mathrm{r}}^{\mathrm{S}}(\mathrm{h})$ demand of those present in the school (pupils, teachers, employees);

$\mathrm{d}_{\mathrm{E}, \mathrm{r}}^{\mathrm{D}}(\mathrm{h})$ demand of those present in the weak user category,

The demand of those present for the resident category in the generic zone can be specified as:

$$
d_{E, r}^{R}(h)=f\left(n_{r}^{R}(h), m_{E}^{R}(h)\right)
$$

with

$\mathrm{n}_{\mathrm{r}}^{\mathrm{R}}(\mathrm{h}) \quad$ resident number in zone $\mathrm{r}$;

$\mathrm{m}_{\mathrm{E}}^{\mathrm{R}}(\mathrm{h}) \quad$ generation coefficient computed according to:

$$
m_{E}^{R}(h)=f\left(P^{2} L_{r}, P S R_{r}, P P_{r}, P C_{r}, \alpha_{F L}(h), \alpha_{S R}(h), \alpha_{P}(h), \alpha_{C}(h)\right)
$$

with

$\mathrm{PFL}_{\mathrm{r}} \quad$ worker percentage in zone $\mathrm{r}$;

$\mathrm{PSR}_{\mathrm{r}} \quad$ student percentage in zone $\mathrm{r}$;

$\mathrm{PP}_{\mathrm{r}} \quad$ retired percentage in zone $\mathrm{r}$;

$\mathrm{PC}_{\mathrm{r}} \quad$ housewife percentage in zone $\mathrm{r}$;

$\alpha_{\mathrm{FL}}(\mathrm{h}), \alpha_{\mathrm{SR}}(\mathrm{h}), \alpha_{\mathrm{P}}(\mathrm{h}), \alpha_{\mathrm{C}}(\mathrm{h})$ calibrating parameters.

The percentage is related to the total number of those present in the study area in the reference period $h$.

The demand of those present in the other categories can be specified as:

$$
\mathrm{d}_{\mathrm{E}, \mathrm{r}}^{\mathrm{k}}(\mathrm{h})=\mathrm{f}\left(\mathrm{m}_{\mathrm{E}}^{\mathrm{k}}(\mathrm{h}), \mathrm{n}_{\mathrm{r}}^{\mathrm{k}-}(\mathrm{h})\right)
$$

where

$\mathrm{m}_{\mathrm{E}}^{\mathrm{k}}(\mathrm{h})$ is a calibrating parameter, with $\mathrm{k}$ equal to:

W for employees;

C for occasional customers;

S for schools;

D for weak users; 
$\mathrm{n}_{\mathrm{r}}^{\mathrm{k}-}(\mathrm{h})$ is the number of users of category k- in zone $\mathrm{r}$, with k- category index; it is worth pointing out that the index $\mathrm{k}$ - represents a different notation from that previously considered for $\mathrm{k}$, because the demand in the occasional customer category is specified as a function of employee number. In particular, $\mathrm{k}$ - is equal to:

W for employees and occasional customers;

S for schools;

D for weak users;

Demand of those willing to evacuate can be specified according to a behavioural approach, considering random residuals i.i.d. according to a Gumbel variable.

The model is generic, for all categories. We assume the absence of targets and:

- the citizen user as the decision-maker;

- a choice set including the alternative to evacuate or not to evacuate;

- attributes and parameters related to socio-economic properties and alternative specific attributes (ASA).

In the following we propose an example of specification of systematic utility for the alternatives:

with

$$
\begin{gathered}
\mathrm{V}_{\text {evacuate }}=\mathrm{f}(\text { Women, W_PU, DL, CO, Centre }) \\
\mathrm{V}_{\text {not_evacuate }}=\mathrm{f}(\mathrm{R}, \text { NOFL })
\end{gathered}
$$

Women dummy equal to 1 for women, 0 otherwise;

$\mathrm{R}$

NOFL dummy equal to 1 for the unemployed, 0 otherwise;

W_PU dummy equal to 1 for those employed in the public sector, 0 otherwise;

Centre dummy equal to 1 if the origin is in central zone, 0 otherwise;

DL dummy equal to 1 for users with a driving licence, 0 otherwise;

$\mathrm{CO}$ dummy equal to 1 for users owning vehicles, 0 otherwise.

\subsection{Specification of a joint model simulating modal choices and distribution}

Given an emergency scenario, the modal choice model simulates the number of people using a transport mode from a certain origin to a certain refuge area; the distribution model simulates the probability of trips undertaken by people going to a certain refuge area, given departure from zone $\mathrm{r}$ in period $\mathrm{h}$.

In this work we consider a model which simulates modal choice jointly with distribution: these are two simultaneous choices. These choices are supposed targeted for school staff and weak user categories. For the remaining categories we suppose the absence of targets, the citizen user as the decision-maker and a behavioural approach, with random residuals i.i.d. according to a Gumbel random variable of zero mean and parameter $\theta$.

Moreover we suppose:

- a choice set which includes the alternatives: pedestrian with a fixed refuge area (cf,pedestrian), pedestrian with a non-fixed refuge area (ncf,pedestrian), car with a fixed refuge area (cf,car); 
- $\quad$ attributes and parameters related to level of service.

The systematic utility for the alternatives will be specified as:

$$
\begin{aligned}
& \mathrm{V}_{\text {cf,pedestrian }}=\mathrm{f}\left(\mathrm{TP}_{\mathrm{r}, \mathrm{cf}}\right) \\
& \mathrm{V}_{\text {ncf,pedestrian }}=\mathrm{f}\left(\mathrm{TP}_{\mathrm{r}, \mathrm{ncf}}\right) \\
& \mathrm{V}_{\text {ncf,pedestrian }}=\mathrm{f}\left(\mathrm{TS}_{\mathrm{r}, \mathrm{cf}}\right)
\end{aligned}
$$

with

$\mathrm{TP}_{\mathrm{r}, \mathrm{cf}}$ time on the pedestrian network from origin $\mathrm{r}$ to fixed refuge area $\mathrm{c}$;

$\mathrm{TP}_{\mathrm{r}, \mathrm{ncf}}$ time on the pedestrian network from origin $\mathrm{r}$ to non-fixed refuge area ncf;

$\mathrm{TS}_{\mathrm{rc}}$ time on the road network from origin $\mathrm{r}$ to fixed refuge area $\mathrm{c}$.

\section{Software for demand model calibration}

In this section we describe some software used for demand model calibration: Alogit [15], HieLoW [16], and software to calibrate mixed multinomial logit model [6].

Alogit exploits the ability of logit models to explain and predict many aspects of consumer behaviour, the main variables determining the choices made by consumers and allowing forecasts of future choices. To help in the specification, estimation, improvement and application of logit models, Alogit is organized to perform three main functions:

- Prepare: the logit model is set up and the data are prepared and checked;

- Estimate: unknown coefficients appearing in the model are estimated from the data;

- Apply: the model is tested and/or applied for forecasting.

The Prepare functions are to specify the model and to amend, check and prepare the data for subsequent analysis. By specification we mean the definition of the utility functions for each of the alternatives. The data are input from the original base data file to Prepare. They may then be amended by transformations specified by the user. The transformed data are then checked to ensure their suitability for logit modelling and written out in a special compressed format to a temporary file for use by Estimate.

The Estimate function is to estimate the values of the parameters appearing in the utility functions. This is an iterative process, which is repeated until the step taken becomes smaller than a defined test value. The resulting parameters are stored and a report is given of the results of the estimation.

The Apply function can be used to test the quality of the model that has been estimated or to make forecasts. The main tests that can be made are to apply the model under unchanged circumstances and compare the results obtained with what was actually observed in the data.

The last version of Alogit allows the parameter calibration with revealed preference or stated preference data.

HieLoW is software designed to calibrate parameters in discrete choice models. It allows a multinomial or a hierarchical (nested) logic model to be estimated. To improve the quality of estimated models, HieLoW provides the analyst with detailed statistical information. Based on recently developed trustregion methods, the maximization algorithm of HieLoW explicitly exploits, when needed, the non-concavity of the loglikelihood function. HieLoW is 
integrated in the WindowsTM environment. A permanent dialogue, through dedicated boxes, leads the analyst from model specification up to results analysis. A tutorial helps beginners get familiar with HieLoW. A glossary and a permanent contextual help system are also included to facilitate the user's work.

Mixed logit (also called random-parameters logit) generalizes standard logit by allowing the parameter associated with each observed variable to vary randomly across customers. The moments of the distribution of customerspecific parameters are estimated. Variance in the unobserved customer-specific parameters induces correlation over alternatives in the stochastic portion of utility. As a result, mixed logit does not exhibit the restrictive forecasting patterns of standard logit. The software realized by Train [7] includes the following files:

- mxlmsl.m is the code that the user runs; the user specifies the model within this code;

- doit.m is a script that is called up at the end of mxlmsl.m; it checks the data, transforms the data into a more useful form, performs the estimation and prints results;

- check.m is a function that checks the input data and specifications; it provides error messages and terminates the run if anything is found to be incorrect;

- $\log$ lik.m is a function that calculates the log-likehood function and its gradient; this function is input to Matlab's fminunc command, which is part of Matlab's Optimization Toolbox; this function calls llgrad2.m;

- $\quad$ llgrad2.m is a function that calculates for each person the probability of the chosen alternatives and the gradient of the log of this probability;

- der.m is a function that calculates the derivative of each random coefficient with respect to the model parameters;

- makedraws.m is a function that creates the standardized draws that will be used in the run, based on the specifications given by the user in mxlmsl.m;

- trans.m is a function that transforms the standardized draws into draws of coefficients;

- data.txt is an ascii file of data on vehicle choice; the data and its format are described within mxlhb.m;

- myrunKT.out is the output file of running maxlhb.m with no modifications. This software allows parameter calibration considering RP and SP surveys [17].

\section{DSS for demand model evaluation}

Several commercial Decision Support Systems (DSS) are available to evaluate transport demand. Generally, these belong to the GIS (Geographic Information Systems) software class. GIS software integrates maps with their respective information or attributes. Through its ability to link spatial data (maps) and nonspatial data (attribute information) in one location, GIS provides a framework for efficient data storage and data retrieval, intuitive display of information in a spatial context, and combining various types of information so that the data may be analyzed further. 
Referring to demand model evaluation, GIS can be subdivided into two main classes:

- generic GIS software, developed and implemented in several fields (marketing, planning, business analysis, transport, and so on);

- transportation GIS software, developed and implemented for specific transportation problems.

Among the software belonging to the first class, we recall:

- ArcInfo [18], the first GIS software available on the market;

- MapInfo [19], a powerful Microsoft Windows-based mapping and geographic analysis application from experts in location intelligence.

The software belonging to the second class includes:

- Omnitrans [20], which provides a versatile working environment for multimodal transport planning and modelling; it offers an integrated software platform for visual display of models and graphical presentation of results, strong project management tools to assist in managing all of the information associated with model scenarios;

- TransCAD [21], designed specifically for use by transportation professionals to store, display, manage, and analyze transportation data; TransCAD combines GIS and transportation modelling capabilities in a single integrated platform, providing capabilities that are unmatched by any other package; TransCAD can be used for all modes of transportation, at any scale or level of detail; TransCAD provides:

- a powerful GIS engine with special extensions for transportation;

- mapping, visualization and analysis tools designed for transportation applications;

application modules for routing, travel demand forecasting, public transit, site location and area management;

- Emme/2 [22] is a graphical software tool for multimodal transportation planning, which allows the transportation network to be modelled and assigns the traffic generated under a given set of conditions;

- PTV [23] is a software suite for transportation planning and operation analyses.

DSS belonging to the second class generally include comprehensive tools for trip generation, trip distribution and mode split modelling. The goal of trip generation is to estimate the number of trips, by purpose, that are produced or originate in each zone of a study area. Trip generation is performed by relating frequency of trips to the characteristics of the individuals, the zone and the transportation network. In some cases, there are two primary tools for modelling trip generation:

- Cross-Classification, which separates the population in an urban area into relatively homogeneous groups based on certain socio-economic characteristics; average trip production rates per household or individual are then empirically estimated for each classification;

- Regression Models, which allow evaluation and application of multivariable aggregate zonal models and disaggregate models at the household or individual level. 
Trip distribution models are used to predict the spatial pattern of trips or other flows between origins and destinations. DSS provide numerous tools with which to perform trip distribution, including procedures to implement growth factor methods, apply previously-calibrated gravity models, generate friction factors and calibrate new model parameters.

Mode choice models are used to analyze and predict the choices that individuals or groups of individuals make in selecting the transportation modes that are used for particular types of trips. Typically, the goal is to predict the share or absolute number of trips made by mode. Software provides procedures for calibrating and applying mode choice models based on multinomial and nested logit models, and may be pursued at either a disaggregate or aggregate zonal level. Estimation of the parameters in the nested logit and multinomial logit model is performed by the method of maximum likelihood, which calculates the set of parameters that are most likely to have resulted in the choices observed in the data.

\section{Conclusions}

In this paper, in the context of emergency conditions, a generation model and a joint model simulating modal choice and distribution was presented. All the models were calibrated using SP and RP data; the latter are considered similar to SP with physical verification. Software for demand model calibration and estimation was analyzed.

The proposed model was calibrated using the data obtained from a real experiment in the urban area of Melito Porto Salvo (Italy) in the framework of the SICURO research project $[9,10]$. We performed pre-trial and trial evacuation. For users of each evacuation zone we conducted RP and SP surveys [24]. For the test description, refer to [9] and [24]. Data were recorded and developed by laboratory analysis to calibrate the proposed model. In order to maximize the likelihood function we implemented Alogit [15] software.

In summary, as regards the generation model, the user category with the least probability of being in the given evacuation area was the resident category. As regards willingness to participate in evacuation, this amounted to about $40 \%$ for every user category. In relation to the modal split with distribution model, the alternative with the greatest probability concerns the choice of refuge area fixed by the mayor, with car mode.

\section{References}

[1] Domencich T.A., McFadden D. Urban travel demand: a behavioural analysis, American Elsevier, New York, 1975.

[2] Ben Akiva M., Lerman S., Discrete choice analysis: theory and application to travel demand, MIT Press, Cambridge, MA, 1985.

[3] Train, K. Discrete choice methods with simulation, MIT Press, Cambridge, MA, 2003.

[4] McFadden D.L., Modelling the choice of residential location. In Interaction Theory and Planning Models, 75-96, North Holland, Amsterdam, 1978. 
[5] Daganzo C.F., Multinomial Probit: the theory and its application to demand forecasting, Academic Press, New York, 1979.

[6] McFadden, D.L., Train, K.: Mixed MNL models for discrete response. In Journal of Applied Econometrics, 15, 447-470, 1996.

[7] F. Russo, A. Vitetta, The road network design problem to improve the safety during exogenous flow perturbations, Proceedings of the $29^{\text {th }}$ ISATA Conference, Florence, 1996.

[8] F. Russo, A. Vitetta, Risk in anthropic environments: methodologies for risk evaluation and exposition reduction. In Environmental Health Risk III, Brebbia et al., WIT Press, Boston, 2005.

[9] Russo, F., Chilà, G., Domanda di trasporto in condizioni di emergenza. Modelli e metodi per la simulazione, applicazione ad un caso reale, Franco Angeli, Milan, 2007.

[10] Russo, F., Chilà, G., Safety of users in road evacuation: demand models, Urban Transport XIII, Brebbia C.A. (ed.), WIT Press, 773-782, and in Urban Transport: Safety of Users in Road Evacuation, Russo F. (ed.), WIT Press, 11-20, 2007.

[11] Russo, F., Vitetta, A., Security and Safety in transportation system: models for evacuation analysis in emergency conditions. In International Journal of Sustainable Development and Planning, vol.1, issue 2, 2006.

[12] Wilmot C.G., Fu H., Static vs. Dynamic and Aggregate vs. Disaggregate: A Comparison Between Practice and Research in Hurricane Evacuation Travel Demand Modeling. In Transp. Research Board 86th Annual Meeting, 2007.

[13] Wilmot C.G., Fu H., A sequential logit dynamic travel demand model for hurricane evacuation. In Transp. Research Record, Vol.1882, 19-26, 2004.

[14] Ortuzar, J. de D., Willumsen, L.G., Modelling Transport, John Wiley \& Sons Ltd, Chichester, 2006.

[15] Daly, A., Alogit 3.2 User's Guide, The Hague: Hague Consulting Group, 1992.

[16] Bierlaire, M. (1995) http://roso.epfl.ch/mbi/HieLoW.html

[17] Train, K., Wilson, W.W. (2007) Estimation on Stated-Preference Experiments Constructed from Revealed-Preference Choices. http://elsa.berkeley.edu/ train/spoffrp.pdf

[18] ArcInfo (2009) http://www.esri.com/software/arcgis/arcinfo/index.html

[19] MapInfo (2009) http://www.mapinfo.it/location/integration

[20] OmniTrans (2009) http://www.omnitrans-international.com/

[21] TransCAD (2009) http://www.caliper.com/tcovu.htm

[22] Emme/2 (2009) http://www.inro.ca/en/index.php

[23] PTV (2009) http://ptvamerica.com/docs/VISION2007.pdf

[24] Russo, F., Chilà, G. (2008) Safety of users in road evacuation: RP vs. SP surveys in demand analysis. In Urban Transport XIV, Brebbia C.A. (ed.), WIT Press, 703-713. 